Gynäkol Geburtsh Rundsch 1994;34(Suppl 1):45

\title{
Aktinomykose und Adnexitis
}

\begin{tabular}{|l|l|l|}
\hline K. & Reisenberger \\
\hline B. & & Sternberger \\
\hline S. & & Vogl \\
\hline Ch. & Egarter \\
\hline
\end{tabular}

Univers.-Frauenklinik Wien, Abteilung Geburtshilfe und Gynäkologie (Vorst.: Prof. Dr. P. Husslein)

Dr. K. Reisenberger, Univers.-Frauenklinik, Spitalgasse 23, A-1090 Wien

Aktinomykose ist eine Infektionskrankheit die durch Actino-myces israeli, ein Grampositives anaerobes Stäbchen, hervorgerufen wird. Die genitale Manifestation einer AktinomykoseInfektion stellt ein relativ seltenes Ereignis dar und zeigt eine eindeutige Korrelation mit der Verwendung eines IUD zur Kontrazeption. Einer der zusätzlichen Risikofaktoren scheint eine anaerobe Keimflora der Vagina zu sein. Die Diagnose einer Besiedelung des Genital-traktes, die noch nicht gleichzusetzen ist mit einer definitiven Infektion, findet sich meist als Zufallsbefund bei zytologischen Abstrichen im Rahmen einer Routineuntersuchung. Die konservative Therapie der Wahl ist die Behandlung mit Penicillin, bei abszendierenden In-fektionen steht die chirurgische Sanierung des Entzündungsherdes, unter entsprechender antibiotischer Abschirmung, im Vordergrund.

Erreger

Actinomyces israeli ist ein Gram-positives anaerobes Stäbchen das zur Gruppe der Aktinomyceten gerechnet wird. Die Kolonien des Erregers werden als Drusen bezeichnet und können schon ma-kroskopisch als gelbliche, körnige Gebilde mit einer Größe bis zu 2 mm erkannt werden. Am häufigsten besiedelt der Erreger die Mundhöhle als harmloser Kommensal und führt bei Gewebsverlet-zung zur zervikofacialen Aktinomykose. Der Nachweis gelingt mit Färbepräparaten, (Gram-, Hämatoxylin-Eosin oder PAP) oder durch kulturelle Anzüchtung unter anaeroben Bedingungen auf Spezialnährböden, wobei hier allerdings erst nach drei bis vier Wochen ein positives Ergebnis zu erwarten ist.

Genitale Aktinomykose

Die asymptomatische Besiedelung des Genitaltraktes, wird vor allem bei Frauen gefunden, die ein IUD zur Kontrazeption tragen. Über die Häuñgkeit gibt es sehr unterschiedliche Angaben. Pettiti und Mitarbeiter konnte in einer retrospektiv durchgeführten Unter-suchung bei 80.000 Papanicolaou-Abstrichen 107 Aktinomykose-positive Abstriche nachweisen. Die Koinzidenz zwischen Aktinomykose und IUD wird auch in dieser Untersuchung bestätigt, da nur drei der 107 positiven Fälle kein IUD trugen.

Bei einem Teil der symptomlosen Trägerinnen kommt es zu einer Aszension dieses Keimes in den oberen Genitaltrakt, dabei spielen

anaerobe Erreger eine Leitfunktion. Aktinomyceten können an den Tuben tuberkuloseähnliche, tumorartige, später nekrotisierend, ein-schmelzende Affektionen bilden. Der Verlauf ist chronisch mit einer Tendenz zur Abszeß- und Fistelbildung. Tubovarialabszesse, die sich 
manchmal unter dem sonographischen Bild eines Ovarialtumors zeigen, sind ebenso als Komplikation beschrieben wie Affektionen von Seiten des Darmes im Sinne einer Ileussymptomatik. In seltenen Fallen kann es auch zu einer tumorähnlichen Metastasierung in an-dere Organsysteme, sowie zu Fistulierungen durch die Haut kom-men.

Therapie

Die Therapie der Wahl bei Aktinomykose besteht in einer Langzeittherapie mit Penicillin-G in der Dosierung von 10-20 Mil-lionen Einheiten für 4-6 Wochen. Anschließend soil eine Nachbehandlung mit Penicillin V für zumindest zwei Monate erfolgen. Al-ternativ dazu können bei Penicillinallergie auch Tetracycline bzw. Clindamycin verabreicht werden. Die früher empfohlenen Sulfo-namide verbessern auch in Kombination mit Penicillinen die Therapie nicht und zählen deshalb und auch aufgrund ihrer Neben-wirkungen, nicht zur Therapie der ersten Wahl. Die Antibiotika-therapie muß wegen der schlechten Penetration ins Granulations-gewebe über lange Zeit und in hohen Konzentrationen durchgeführt werden.

Besteht eine der oben beschriebenen Komplikationen, wie etwa ein Tubovarialabszeß, so wird die alleinige antibiotische Therapie nicht zum Erfolg führen, sondern es ist wie in der Literatur auch mehrmals beschrieben, eine chirurgische Intervention meist unum-gänglich.

Unklar ist, ob auch asymptomatische Formen der Aktinomykose einer Therapie bedürfen oder ob der Wechsel des IUD als alleinige therapeutische Maßnahme dabei ausreichend ist. Da die Infektion fast immer in Kombination mit einer Anaerobierinfektion vor-kommt, soil auf eine gleichzeitig bestehende Besiedelung der Vagina mit Anaerobiern geachtet werden und diese in die Therapieüber-legungen mit einbezogen werden. Die zusätzliche Gabe eines Anti-biotikums, das das anaerobe Keimspektrum abdeckt erscheint sinn-voll.

\section{Literatur}

Pettiti DB, Yamamoto D. Morgenstern N: Factors associated with actinomyces-like organisms on Papanicolaou smears in users of IUD. AM J Obstet Gynecol 1983; 145: 338. Franz HB. Strohmaier WL, Geppert M, Wechsel H: Infiltrierender Tubovarialabszeß bei IUP assoziierter Actinomykose. Geburts Frauenheilk 1992; 54.

Hoffmann MS, Roberts WS. Salomon P, Gu-nasekarin S, Cavanagh D: Advanced actinomycotic pelvic inflammatory disease simulating gynecologic malignancy. A report of two cases. J Reprod Med 1991; 36 (7): 543-545.

Buchan H, Villard Mackintosh L, Vessey M, Yeates D, Mcpherson K: Epidemiology of pelvic inflammatory disease in parous women with special reference to intrauterine devices use. $\mathrm{Br} \mathrm{J}$ Obstet Gynecol 1991; 97: 780. Simon C. Stille W: Antibiotikatherapie in Klinik und Praxis: Schattauer Verlag 1993. 